\title{
Effect of Mulligan upper cervical manual traction in the treatment of cervicogenic headache: a randomized controlled trial
}

\author{
DOI: https://doi.org/10.5114/pq.2019.87738
}

\author{
Mohamed A. Khalil', Hamed Alkhozamy², Salwa Fadle², Abdelsalam M. Hefny ${ }^{3}$, Mostafa A. Ismail ${ }^{4}$ \\ ${ }^{1}$ Department of Physical Therapy, Alahrar Educational Hospital, Zagazig, Egypt \\ ${ }^{2}$ Department of Orthopaedic Physical Therapy, Faculty of Physical Therapy, Cairo University, Cairo, Egypt \\ ${ }^{3}$ Department of Orthopaedic Surgery, Faculty of Medicine, Zagazig University, Zagazig, Egypt \\ ${ }^{4}$ Egypt Centre for Orthopaedic Manual Physical Therapy, Tanta, Egypt
}

\section{Abstract}

Introduction. Cervicogenic headache (CGH) is a serious condition manifested by upper cervical facet joints dysfunction. Mulligan upper cervical sustained natural apophyseal glide was noted to be effective in CGH but Mulligan upper cervical manual traction (MUCMT) has not yet been investigated. The purpose of the study was to compare the effect of MUCMT vs. traditional treatment (TT) in patients with CGH.

Methods. A randomized controlled prospective parallel single-blind trial was performed. Overall, 30 patients with CGH aged 30-55 years were randomly and equally allocated into the MUCMT group (A) and TT group (B) by using permuted block randomization. Group A participants were treated by TT and MUCMT while group B received hot packs, transcutaneous electrical nerve stimulation, and deep cervical flexors strengthening exercise. Patients gained 3 sessions every week for 3 weeks followed by home exercise for 3 months. Pre-treatment, post-treatment, and follow-up values for all outcome measures were recorded. The primary outcome was headache intensity. Secondary outcomes involved headache frequency, headache duration, neck disability index, and upper cervical rotation range of motion.

Results. Within groups, statistical analysis revealed a significant difference in the comparison of pre- vs. post-treatment and post-treatment vs. follow-up mean values of all outcomes. Between groups, no statistical significance was observed in posttreatment and follow-up data, with 1 exception regarding upper cervical range of motion in favour of MUCMT.

Conclusions. MUCMT is an effective treatment in patients with CGH, mainly with regard to upper cervical rotation range of motion. Key words: cervicogenic headache, neck pain, Mulligan manual cervical traction

\section{Introduction}

Headache is a frequent syndrome, disturbing up to $66 \%$ of people, with an expected lifetime occurrence of $96 \%$; consequently, it harmfully affects both life quality and society productivity [1]. Cervicogenic headache $(\mathrm{CGH})$ is documented to affect $2.5 \%$ of adult individuals and result in $15-20 \%$ of all chronic and frequent headaches [1, 2]. Moreover, in 1983, it was described as a distinct disorder and differentiated from other categories of headaches such as migraine and tension type headache [1, 2]. The pain in CGH initiates from the cervical region and radiates to a part or more in the head and/or the face; in addition, CGH pain is felt in one side, mostly in the occipital area, frontal region, or retro-orbital region [3]. The International Headache Society (IHS) CGH diagnostic criteria depend, at least in part, on a patient's response to diagnostic facet injection blocks. This is not applicable in clinical practice; however, the diagnostic criteria of CGH according to the CGH International Study Group (CHISG) present a readily suitable, effective, and easy method that is useful in manual therapy practice [4].

Fredriksen et al. [5] declared that the updated IHS classification of headache ( $3^{\text {rd }}$ edition, beta version) was still probably not a safe basis for diagnosing CGH but the CHISG criteria might seem safer in $\mathrm{CGH}$ diagnosis. CGH pathophysiology was explained by the convergence between the trigeminal nerve and the upper cervical nerves afferents in the trigemi- nocervical nucleus, which clearly states the mechanism underlying the pain radiation to the head [6]. In this convergence, painful signals from the upper cervical spinal nerves congregate onto second-order neurons that get innervations from nearby cervical nerves and from the first branch of the trigeminal nerve throughout the trigeminal nerve spinal tract. In this way, upper cervical pain radiates to parts of the head such as auricular and occipital regions, which have innervations by cervical nerves [6]. Hall et al. [7] stated that individuals with $\mathrm{CGH}$ commonly had upper cervical articular dysfunction mainly affecting the upper 3 cervical segments; in addition to that, there is impairment of deep neck flexors strength and endurance.

Manual examination can be applied to diagnose CGH through detecting the limited range of motion (ROM) of upper cervical rotation at $\mathrm{C} 1-\mathrm{C} 2$ by using the flexion-rotation test (FRT) [8]. Ogince et al. [9] mentioned that the specificity of FRT equalled $90 \%$, with $91 \%$ sensitivity $(p<0.001)$ and $91 \%$ diagnostic accuracy $(p<0.001)$, Moreover, FRT considerably helps in CGH diagnosis through detecting limited upper cervical rotation ROM. Fernández-de-Las-Peñas and Courtney [10] declared that there were many manual therapies in the management of $\mathrm{CGH}$, but still no superiority of any was achieved and this might be a result of inconclusive understanding of CGH pathology. Mulligan manual therapy approaches have been noted to be significantly valuable in the management of cervical dysfunctions [11]. Buyukturan

Correspondence address: Dr. Mohamed Khalil, Dr. Hamid S. Al-Ahmedy Hospital Co, Second Circle Road with Sayed Al-Shouhda, Almadina Almonawarah, Kingdom of Saudi Arabia, P.O. Box 6476, Code 41441, e-mail: makhalil@utica.edu

Received: 26.06 .2019

Accepted: 16.08 .2019

Citation: Khali MA, Alkhozamy H, Fadle S, Hefny AM, Ismail MA. Effect of Mulligan upper cervical manual traction in the treatment of cervicogenic headache: a randomized controlled trial. Physiother Quart. 2019;27(4):13-20; doi: https://doi.org/10.5114/pq.2019.87738. 
et al. [12] studied the effects of Mulligan sustained natural apophyseal glide approach in the treatment of neck pain in elder patients. The results revealed that Mulligan mobilization group had better outcome measures in pain level, ROM, and daily living activities. Hall et al. [7] mentioned that intermittent traction lengthened all vertically oriented soft tissues, helped open the jammed facet joints and relieved intervertebral disc compression, thus reducing pain of the upper cervical region. The research question concentrates on whether Mulligan upper cervical manual traction (MUCMT) benefits patients with $\mathrm{CGH}$. The study objective was to explore the effect of MUCMT in patients with CGH in terms of headache intensity, headache frequency, headache duration, neck disability index (NDI), and upper cervical rotation ROM to the affected side.

\section{Subjects and methods}

Study design

A randomized, controlled, parallel, assessor-blinded clinical trial was designed to study differences in CGH intensity, frequency, and duration, as well as function and upper cervical ROM in patients with $\mathrm{CGH}$.

\section{Patients and settings}

Overall, 30 patients were enrolled in the clinical study from the outpatient physical therapy clinic of the Faculty of Physical Therapy, Cairo University from May 2017 to April 2018. The selected patients (males and females) were referred by orthopaedic and neurology physicians, diagnosed with CGH, aged $30-55$ years; they had experienced frequent $\mathrm{CGH}$ and cervical pain for at least 3 months. Other inclusion criteria involved unilaterality of pain and limited FRT with at least $10^{\circ}$ less than normal. Headache is referred to as frequent if it has appeared minimally once every week through the recent 3 months $[13,14]$. Exclusion criteria comprised migraine, tension type headache, cluster headache, cervical disc lesion with or without radiculopathy, entrapment neuropathy, myelopathy, rheumatoid arthritis or previous cervical spine surgeries, spinal tumours, dysfunctions in the temporomandibular joint [15].

CHISG major diagnostic criteria (not including diagnostic anaesthetic blockades) were applied for all referred individuals with $\mathrm{CGH}$ diagnosis. The patients (19 women, 11 men) were informed that the recorded data would be submitted for publication. They were educated on how to use a daily headache diary for 1 week and then returned it for baseline examination and data collection. Pre-treatment assessment

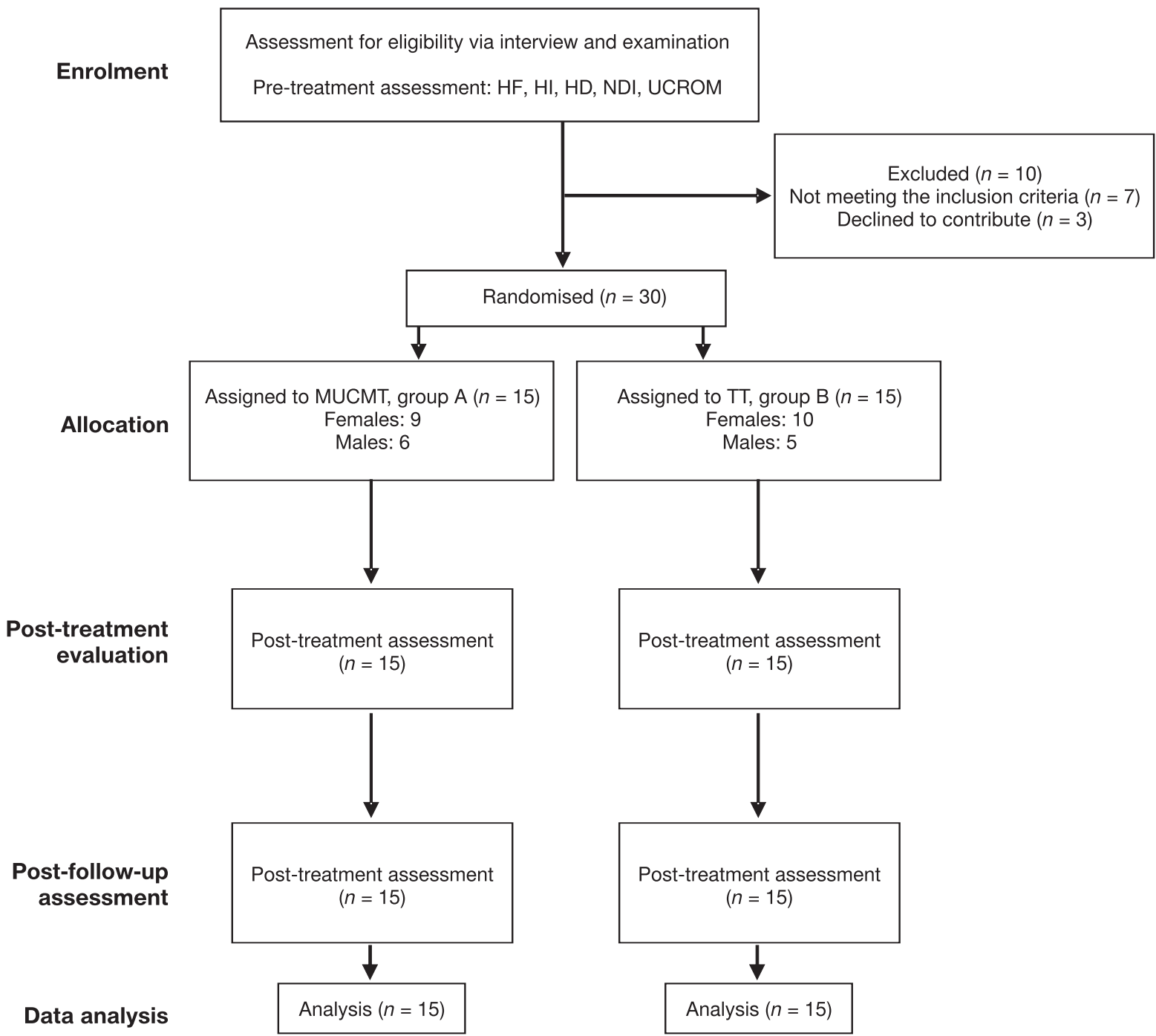

$\mathrm{HF}$ - headache frequency, $\mathrm{HI}$ - headache intensity, HD - headache duration, NDI - neck disability index, UCROM - upper cervical rotation range of motion, MUCMT - Mulligan upper cervical manual traction, TT - traditional treatment

Figure 1. CONSORT flow diagram of participants through the study 
was performed in all participants by a manual physical therapist blinded to the trial protocol. The included patients were randomly assigned to groups $\mathrm{A}$ ( $n=15$ patients; 6 males, 9 females) and B ( $n=15$ patients; 5 males, 10 females) by an independent, blinded research assistant by using permuted block randomization (Figure 1).

\section{Randomization}

Permuted block randomization was applied to decrease possible assortment and unintentional bias, as well as to create a balance in the sample size between groups $A$ and $B$ [16]. A block size of 6 was used and the written blocks were provided in closed envelopes (such as AAABBB, ABABAB, BBBAAA...). Five envelopes were randomly selected by the first patient to be used in the trial to cover 30 patients. One envelope was then randomly chosen by the first patient and then the following 5 patients were distributed as pointed by the order of the selected envelope sequence. The process was repeated until the required number in each group was reached.

\section{Treatment provider}

The treatment was provided by a clinical orthopaedic manual physical therapist who was a certified Mulligan practitioner and had been applying the approach for 8 years. The treatment provider was not blinded to the treatment protocols.

\section{Assessment procedure}

A daily headache diary was used to report headache parameters (intensity, frequency, and duration) throughout 1 week before baseline assessment, 1 week post-treatment, and 1 week after 3 months as the time of follow-up assessment. Regarding the headache intensity and duration, their mean values were documented and used in the statistical analysis. NDI assessment and upper cervical ROM were recorded at the initial assessment, 1 week post-treatment, and 1 week after the 3 months of follow-up.

1. Headache intensity was measured with a numerical pain rating scale. The patients were asked to indicate the numeric value on an 11-item scale $(0-10)$ that best indicated their headache intensity, where 0 stood for no pain and 10 implied the worst pain [17]. The mean of the registered values was used in the statistical analysis.

2. Headache frequency indicates the mean number of headache attacks the patient suffered through the week.

3. Duration of headache attacks was registered by each patient in the daily headache diary for 1 week. The mean of the values was submitted for statistical analysis.

4. Any limitation in the upper cervical rotation ROM was detected by using FRT with the help of a cervical ROM (CROM) device (manufactured by Performance Attainment Associates, Roseville, MN, USA). The patient lay supine with fully flexed cervical spine to limit the movement of lower cervical segments and to concentrate the movement on $\mathrm{C} 1-\mathrm{C} 2$ (Figure 2). The upper cervical rotation ROM was documented to be $44^{\circ}$ to each side [9]. Patients with $\mathrm{CGH}$ associated with $\mathrm{C} 1-\mathrm{C} 2$ dysfunction show $10^{\circ}$ less than normal upper cervical rotation ROM to the affected side [18]. According to Hall et al. [7], during FRT, the CROM device is fixed over the patient's head, the patient lying supine, shoulders level at the edge of the couch. The body in relaxed end-range cervical spine and upper thoracic spine flexion. The examiner's abdomen supports the head of the participant in flexed

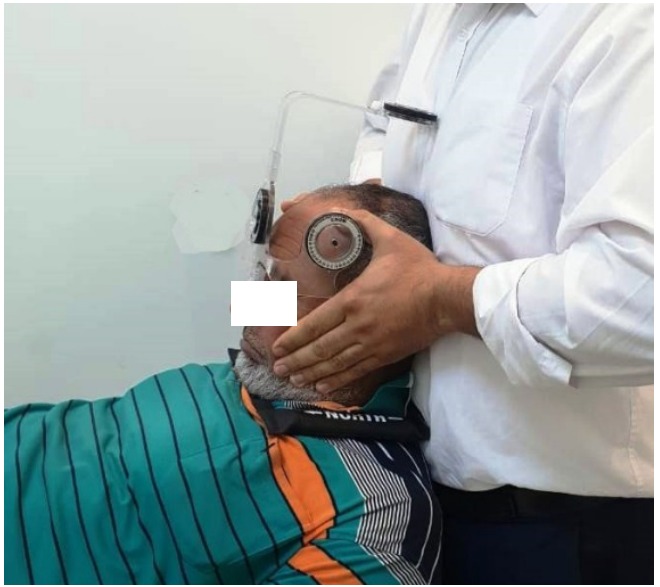

Figure 2. Cervical range of motion device and flexion-rotation test starting posture

position and faces the patient's feet. The therapist keeps the end-range cervical spine flexion with hand contact on each side of the mandible in addition to forward pressure applied by the abdomen of the therapist. The therapist rotates the patient's head to the right and to the left, and the available range is documented. End-range flexion is important to apply the test. The rotation of the head is as pure as possible and no lateral flexion is allowed. In the case of $\mathrm{CGH}$, the end-point is either resistance or pain, whichever comes first.

5. The Arabic version of NDI questionnaire consists of 10 sections, which cover both the patient's symptoms and daily living activities [19, 20].

\section{Treatment procedure}

The treatment protocol was applied for 9 sessions every other day. Group B underwent traditional treatment (TT), which includes hot packs, transcutaneous electrical nerve stimulation (TENS), and deep cervical flexors strengthening exercise. Group A received TT in addition to MUCMT. It was recommended to use superficial heat to gain muscle relaxation before cervical traction for the best effect [21]. The applied TENS machine was Gymna 400, 2 channels, 2-200 Hz frequency range, 10-250 $\mu$ s pulse duration range, and 0-100 mA power range. A conventional TENS with $50-\mathrm{Hz}$ frequency and a pulse duration of $100 \mu$ s was used in our study. TENS was applied to the suboccipital region for 20 minutes [12, 22].

Deep cervical strengthening exercises were performed by using an uninflated pressure sensor biofeedback unit

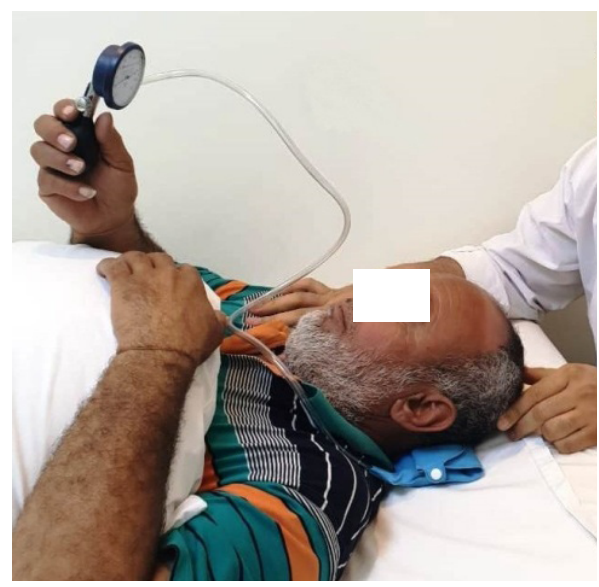

Figure 3. Strengthening deep cervical flexors by using a pressure biofeedback unit 


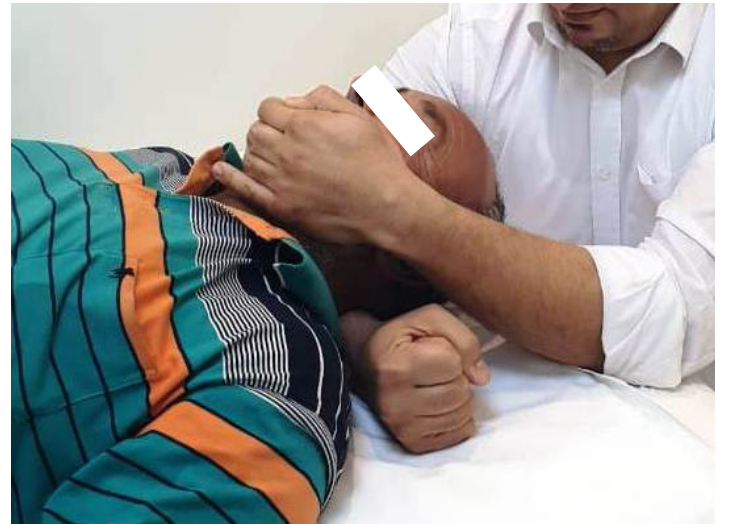

Figure 4. Starting position for Mulligan upper cervical manual traction

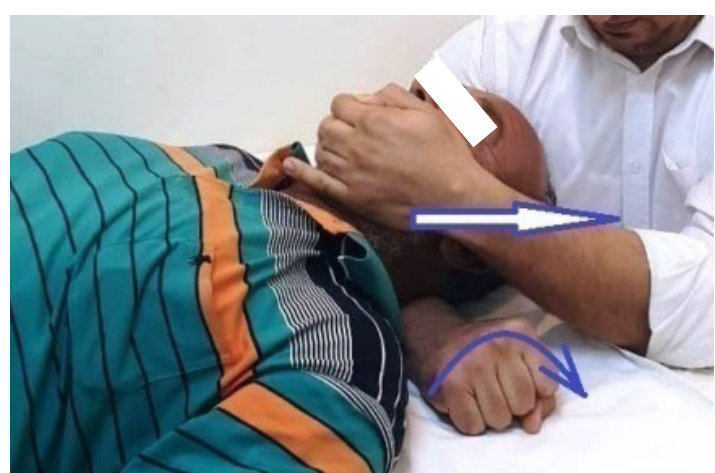

Figure 5. A Mulligan upper cervical manual traction component

(manufactured by Chattanooga group, Hixson, TN, USA), positioned below the neck of the patient in crook lying. The therapist inflated the biofeedback unit up to $20 \mathrm{~mm} \mathrm{Hg}$, which is sufficient to support the neck without making cervical lordosis (Figure 3) [23]. The patient contracted the deep cervical flexors through nodding motions and kept the pressure at $22 \mathrm{~mm} \mathrm{Hg}$; they were asked to keep steady contraction for 10 seconds and this was repeated 10 times with 15-second breaks between contractions in each session [2, 24].

Patients in group A were treated with TT in addition to MUCMT. They lay supine with the neck in neutral posture. The physiotherapist placed the proximal part of his forearm under the cervical spine so that the radial border was tucked under the base of the occipital bone. The fingers of the other hand were placed over the patient's chin (Figure 4). The therapist applied traction through pronating the forearm and simultaneously imposed pressure over the chin to provide a translator component so that it was a combination of translation and rotation of the forearm (Figure 5). There should be no extension of the cervical spine while performing traction as it would lead to jamming of the facet joints. Traction should applied along the spine axis. The force applied to the occiput and chin was equal. The traction force was main- tained for 10 seconds and repeated 10 times every session $[25,26]$.

Additionally, all patients underwent a home exercise program, which included strengthening of deep cervical flexors in the form of 10-second hold of chin in exercises followed by 10 -second relax, repeated 10 times without using any pressure sensor at home. This was applied twice a day up to the follow-up assessment.

\section{Statistical procedures}

The SPSS for Windows software, version 23 (SPSS, Inc., Chicago, IL, USA) was used to conduct the statistical analysis. Two independent variables were included in the test. Firstly, the tested group between subjects factor with 2 levels; group A receiving $T T$ (hot packs, TENS, deep cervical flexors strengthening exercise) in addition to MUCMT, and group B receiving the same TT. Secondly, the measuring periods; within-subject factor which had 3 levels (pre-treatment, post-treatment, and follow-up). Moreover, the test analysed 5 involved dependent variables (headache intensity, headache frequency, headache duration, NDI, upper cervical rotation to the affected side). Accordingly, a $2 \times 3$ mixed MANOVA test was conducted in the comparison of the tested variables of interest at the determined measuring periods in both groups. The initial alpha level was set at 0.05 .

\section{Ethical approval}

The research related to human use has complied with all the relevant national regulations and institutional policies, has followed the tenets of the Declaration of Helsinki, and has been approved by the Research Ethical Committee, Faculty of Physical Therapy, Cairo University (No. P.T.REC/012/001561). The trial was registered in the Pan African Clinical Trials Registry (No. PACTR201706002316214).

\section{Informed consent}

Informed consent has been obtained from all individuals included in this study.

\section{Results}

The between-group comparison by independent $t$-tests indicated no significant differences $(p>0.05)$ in the mean data of age, body mass, patients' height, or history of headache between the tested groups (Table 1).

As presented in Tables 2 and 3, the MANOVA and multiple pairwise comparison tests (post-hoc tests) revealed that in both groups there was a significant decrease $(p<0.05)$ of headache intensity, headache frequency, headache duration, and NDI, as well as a significant increase $(p<0.05)$ in upper cervical rotation ROM to the affected side. These data refer to the following measurements: pre-treatment vs. post-treatment, pre-treatment vs. follow-up, and post-treatment vs.

Table 1. Physical characteristics of patients in both groups

\begin{tabular}{|l|c|c|c|c|}
\hline \multirow{2}{*}{ Items } & Group A & \multicolumn{2}{|c|}{ Comparison } \\
\cline { 3 - 5 } & Agean $\pm S D)$ & Group B & t-value & $p$-value \\
\hline Body mass $(\mathrm{kg})$ & $42.53 \pm 7.15$ & $41.6 \pm 6.62$ & 0.371 & 0.713 \\
\hline Height $(\mathrm{cm})$ & $95 \pm 16.93$ & $87.06 \pm 17.75$ & 1.252 & 0.221 \\
\hline History of headache $($ years $)$ & $173.33 \pm 13.09$ & $168.13 \pm 9.34$ & 1.252 & 0.221 \\
\hline
\end{tabular}


Table 2. Descriptive statistics and $2 \times 3$ mixed design MANOVA for primary and secondary outcome measures in different measuring periods for group A

\begin{tabular}{|l|c|c|}
\hline Measure & $\begin{array}{c}\text { Pre-treatment } \\
(\text { mean } \pm S D)\end{array}$ & $\begin{array}{c}\text { Post-treatment } \\
(\text { mean } \pm S D)\end{array}$ \\
\hline Headache intensity (NPRS score) & $5.33 \pm 1.29$ & $2.8 \pm 0.86$ \\
\hline Headache frequency (number per week) & $3.06 \pm 1.57$ & $1.73 \pm 0.70$ \\
\hline Headache duration (hours/day) & $3.93 \pm 1.38$ & $1.86 \pm 0.83$ \\
\hline Neck disability index & $46.13 \pm 8.76$ & $25.33 \pm 6.66$ \\
\hline Upper cervical rotation ROM (degrees) & $26.73 \pm 5.75$ & $40.86 \pm 3.56$ \\
\hline
\end{tabular}

$S D$ - standard deviation, NPRS - numerical pain rating scale, ROM - range of motion

Table 3. Descriptive statistics and $2 \times 3$ mixed design MANOVA for primary and secondary outcome measures in different measuring periods for group $B$

\begin{tabular}{|l|c|c|}
\hline Measure & $\begin{array}{c}\text { Pre-treatment } \\
\text { (mean } \pm \text { SD) }\end{array}$ & $\begin{array}{c}\text { Post-treatment } \\
\text { (mean } \pm \text { SD) }\end{array}$ \\
\hline Headache intensity (NPRS score) & $5.26 \pm 1.38$ & $3 \pm 1.1$ \\
\hline Headache frequency (number per week) & $2.93 \pm 1.16$ & $1.93 \pm 0.79$ \\
\hline Headache duration (hours/day) & $3.2 \pm 1.26$ & $1.93 \pm 0.70$ \\
\hline Neck disability index & $43.2 \pm 7.62$ & $26 \pm 6.18$ \\
\hline Upper cervical rotation ROM (degrees) & $29.33 \pm 2.38$ & $34.86 \pm 3.06$ \\
\hline
\end{tabular}

$S D$ - standard deviation, NPRS - numerical pain rating scale, ROM - range of motion

Table 4. Multiple pairwise comparisons (post-hoc tests) among different measuring periods for primary and secondary outcome measures in group A vs. group B

\begin{tabular}{|l|c|c|c|}
\hline \multirow{2}{*}{ Measure } & \multicolumn{2}{|c|}{$p$-value (group A vs. group B) } \\
\cline { 2 - 4 } & Pre-treatment assessment & Post-treatment assessment & Post-follow-up assessment \\
\hline Headache intensity (NPRS score) & 0.893 & 0.724 \\
\hline Headache frequency (number per week) & 0.794 & 0.473 \\
\hline Headache duration (hours/day) & 0.141 & 0.815 \\
\hline Neck disability index & 0.337 & 0.779 \\
\hline Upper cervical rotation ROM (degrees) & 0.117 & 0.053 \\
\hline
\end{tabular}

NPRS - numerical pain rating scale, ROM - range of motion, * significant at the alpha level of $<0.05$

follow-up. The significant difference is in favour of the posttreatment and follow-up assessment in comparison with pretreatment and in favour of the follow-up assessment as compared with post-treatment.

As shown in Table 4, multiple pairwise comparison tests (post-hoc tests) for headache intensity revealed no significant differences in the mean pre-treatment, post-treatment, or post-follow-up values between the groups $(p>0.05$ for all comparisons). The same applies to headache frequency, headache duration, and NDI. However, as for upper cervical rotation $\mathrm{ROM}$ to the affected side, although the mean pretreatment values showed no significant differences between the groups $(p>0.05)$, there were significant differences of the mean values in the post-treatment and post-follow-up tests between the groups $(p<0.05)$. This significant increase was in favour of group $A$ as compared with group $B$.

\section{Discussion}

The current study revealed a significant effect in group $A$ and group $B$ in all the post-treatment and post-follow-up values of the outcome measures. Between-group statistics stated no significant differences between the groups in any measured outcomes in post-treatment or in post-follow-up results; the one exception was a significant difference in upper cervical rotation ROM to the affected side in the post-treatment and post-follow-up assessment in favour of group A.

According to our knowledge, this study is the first one to assess the therapeutic effect of MUCMT in patients with $\mathrm{CGH}$. However, the limited previous research regarding this topic allows to compare the results of this trial with similar studies on manual treatments of $\mathrm{CGH}$ and cervical pain. Our results report a significant improvement in CGH intensity, frequency, and duration, as well as NDI and upper cervical ROM to the affected side in both post-treatment and post-follow-up assessments in group $A$. The achieved outcomes were possibly due to the effect of upper cervical traction, which was documented to separate the cervical segments, mobilize the facet joints, relax the cervical muscles, and widen the intervertebral foramina [27]. Our results are supported by some previous studies. Islam et al. [28] investigated the effect of moist heat therapy, manual cervical traction in patients with CGH and mentioned a significant improvement in both pain and disability. Elnaggar et al. [21] 
concluded that intermittent cervical traction was significantly effective on cervical pain and brachialgia in terms of pain and ROM. Chiu et al. [29] treated patients with chronic cervical pain using alternating cervical traction; they revealed no significant difference in the post-treatment values of the measured outcomes. Graham et al. [30] conducted a systematic review and found no evidence to support cervical pain treatment using cervical traction owing to study methodological quality.

Regarding the presented group B, this study states a significant reduction in headache intensity, headache frequency, headache duration, and NDI, as well as a significant improvement in upper cervical ROM to the affected side in both posttreatment and post-follow-up assessments. The achieved results may be due to the strengthening exercise, which may permit the musculotendinous proprioceptors to reduce their stretch reflex responses. Moreover, exercise improves blood supply and reduces metabolite increase, which may eliminate myofascial pain [31]. This trial outcomes are similarly declared by some previous studies. Islam et al. [28] investigated the effect of deep neck flexors training in addition to conventional physical therapy treatment in patients with CGH. They observed a significant improvement in pain and disability. Ylinen et al. [2] stated that strengthening exercise was more significantly effective in patients with $\mathrm{CGH}$ than endurance exercise. Borisut et al. [23] studied the effect of strengthening and endurance exercise for cervical muscles in patients with cervical pain and found a significant improvement in all outcome measures. They explained that the results might be due to a rise in neuromuscular effectiveness and enhancement in deep neck flexor control. On the other hand, Gross et al. [32] mentioned in their review a limited evidence regarding the effect of strengthening exercise in the management of cervical pain due to low quality research. Verhagen et al. [33] showed in their meta-analysis uncertain and lacking evidence for the use of strengthening exercises in patients with neck pain.

Comparing groups $\mathrm{A}$ and $\mathrm{B}$ revealed no significant difference regarding the improvement in headache intensity, headache frequency, headache duration, and NDI measures between the groups. However, upper cervical rotation ROM improved significantly in the MUCMT group when compared with the TT group. Our results are supported by Browder et al. [34], who declared that cervical traction in the intermittent mode in addition to manipulation significantly enhanced neck ROM and reduced pain. Buyukturan et al. [12] detected a significant improvement in pain, ROM, and disability using Mulligan manual approaches in cervical dysfunction. Gautam et al. [35] observed that Mulligan manual exercises were significantly better than Maitland techniques and TT in patients with cervical dysfunctions. Ali et al. [36] compared cervical mobilization and cervical traction in the management of patients with non-specified cervical pain. They documented that cervical mobilization more significantly eliminated pain and disability than cervical traction. These results differ from ours because MUCMT may be more localized and specific to the upper cervical region facet joints, ligaments, and muscles, where the dysfunction presents; as a result, pain intensity dropped and functional level improved with no significant difference between our groups. Moreover, the upper cervical rotation ROM to the affected side was significantly greater in the MUCMT group than in the TT group. Our results reflect the underlying pathology of $\mathrm{CGH}$ and specifically the limited upper cervical ROM, which was documented to be an acceptable reason for $\mathrm{CGH}$ [7].
$\mathrm{CGH}$ relates to cervical joint dysfunction; therefore, several studies on $\mathrm{CGH}$ treatment focused on joint mobilization or manipulation [37]. The central theory implies the presence of a lesion that affects the biomechanical function of a joint, which results in proximal and distal dysfunction and symptoms [38]. According to Gibbons and Tehan [39], the indications for manual therapy are stiffness of joints, restricted movements, joint hypomobility, pain relieve, and release of adhesions. Chettri et al. [27] mentioned the following benefits of mobilization: mechanical effect in the form of correcting the joint motion and decrease in the joint restriction through the separation of joint surfaces; connective tissue effects such as dynamic correction; and neurological effects in the form of pain receptors inhibition.

\section{Limitations}

The improvements in both primary and secondary outcome measures in both groups are limited to 3-month follow-up duration in this trial. This is considered a short-term effect, so long-term effects of MUCMT should be studied in the future research. The number of individuals in this study is limited to 15 in each group; consequently, our results cannot be generalized as a treatment protocol and the number of patients should be increased to make the results more applicable in clinical practice. Furthermore, studying $\mathrm{CGH}$ treatment should be based on the duration of illness and symptoms in terms of acute, subacute, and chronic CGH. The control group received TT, which was documented to relieve $\mathrm{CGH}$ pain and improve the functional level, so including a placebo group in future trials is required to detect more precise effects and results.

\section{Conclusions}

The presented trial showed that MUCMT added to TT was more effective than TT alone in increasing the upper cervical rotation ROM to the affected side. Moreover, both treatment protocols revealed a significant improvement in all outcome measures.

\section{Acknowledgments}

The authors acknowledge the huge support from the scholars whose studies were cited and mentioned in our references of this study. We are thankful to everyone who contributed to the presented work.

\section{Disclosure statement}

No author has any financial interest or received any financial benefit from this research.

\section{Conflict of interest}

The authors state no conflict of interest.

\section{References}

1. Jafari M, Bahrpeyma F, Togha M. Effect of ischemic compression for cervicogenic headache and elastic behavior of active trigger point in the sternocleidomastoid muscle using ultrasound imaging. J Bodyw Mov Ther. 2017;21(4):933-939; doi: 10.1016/j.jbmt.2017.01.001.

2. Ylinen J, Nikander R, Nykänen M, Kautiainen H, Häkkinen A. Effect of neck exercises on cervicogenic headache: a randomized controlled trial. J Rehabil Med. 2010;42(4):344-349; doi: 10.2340/16501977-0527.

3. Sedighi A, Ansari NN, Naghdi S. Comparison of acute effects of superficial and deep dry needling into trigger 
points of suboccipital and upper trapezius muscles in patients with cervicogenic headache. J Bodyw Mov Ther. 2017;21(4):810-814; doi: 10.1016/j.jbmt.2017.01.002.

4. Mitchell B, LeFebvre R. Cervicogenic headache: assessment. Portland: University of Western States, College of Chiropractic; 2013.

5. Fredriksen TA, Antonaci F, Sjaastad O. Cervicogenic headache: too important to be left un-diagnosed. J Headache Pain. 2015;16:6; doi: 10.1186/1129-2377-16-6.

6. Bogduk N, Govind J. Cervicogenic headache: an assessment of the evidence on clinical diagnosis, invasive tests, and treatment. Lancet Neurol. 2009;8(10):959-968; doi: 10.1016/S1474-4422(09)70209-1.

7. Hall T, Briffa K, Hopper D. Clinical evaluation of cervicogenic headache: a clinical perspective. J Man Manip Ther. 2008;16(2):73-80;doi:10.1179/106698108790818422.

8. Jull G, Amiri M, Bullock-Saxton J, Darnell R, Lander C. Cervical musculoskeletal impairment in frequent intermittent headache. Part 1: Subjects with single headaches. Cephalalgia. 2007;27(7):793-802; doi: 10.1111/ j.1468-2982.2007.01345.x.

9. Ogince M, Hall T, Robinson K, Blackmore AM. The diagnostic validity of the cervical flexion-rotation test in $\mathrm{C} 1 / 2$ related cervicogenic headache. Man Ther. 2007;12(3): 256-262; doi: 10.1016/j.math.2006.06.016.

10. Fernández-de-Las-Peñas $C$, Courtney CA. Clinical reasoning for manual therapy management of tension type and cervicogenic headache. J Man Manip Ther. 2014; 22(1):44-50; doi: 10.1179/2042618613Y.0000000050.

11. Bosmia KK, Kotwal JR. Comparison between the effectiveness of manual Mulligan traction and intermittent electric traction in cervical spondylosis. IOSR J Nurs Health Sci. 2015;4(5):59-64; doi: 10.9790/1959-04535964.

12. Buyukturan O, Buyukturan B, Sas S, Karartı C, Ceylan I. The effect of Mulligan mobilization technique in older adults with neck pain: a randomized controlled, doubleblind study. Pain Res Manag. 2018;2018:2856375; doi: $10.1155 / 2018 / 2856375$.

13. Uthaikhup S, Sterling M, Jull G. Cervical musculoskeletal impairment is common in elders with headache. Man Ther. 2009;14(6):636-641; doi: 10.1016/j.math.2008.1 2.008 .

14. Sjaastad O, Fredriksen TA, Pfaffenrath V. Cervicogenic headache: diagnostic criteria. Headache. 1998;38(6): 442-445; doi: 10.1046/j.1526-4610.1998.3806442.x.

15. Groeneweg $R$, Kropman $H$, Leopold $H$, van Assen L, Mulder J, van Tulder MW, et al. The effectiveness and cost-evaluation of manual therapy and physical therapy in patients with sub-acute and chronic non specific neck pain. Rationale and design of a randomized controlled trial (RCT). BMC Musculoskelet Disord. 2010;11:14; doi: 10.1186/1471-2474-11-14.

16. Suresh KP. An overview of randomization techniques: an unbiased assessment of outcome in clinical research. J Hum Reprod Sci. 2011;4(1):8-11; doi: 10.4103/09741208.82352.

17. Castarlenas E, Jensen MP, von Baeyer CL, Miró J. Psychometric properties of the numerical rating scale to assess self-reported pain intensity in children and adolescents: a systematic review. Clin J Pain. 2017;33(4):376383; doi: 10.1097/AJP.0000000000000406.

18. Hall T, Robinson $\mathrm{K}$. The flexion-rotation test and active cervical mobility - a comparative measurement study in cervicogenic headache. Man Ther. 2004;9(4):197-202; doi: 10.1016/j.math.2004.04.004.
19. Vernon $\mathrm{H}$. The neck disability index: state-of-the-art, 1991-2008. J Manipulative Physiol Ther. 2008;31(7): 491-502; doi: 10.1016/j.jmpt.2008.08.006.

20. Shaheen AA, Omar MT, Vernon H. Cross-cultural adaptation, reliability, and validity of the Arabic version of neck disability index in patients with neck pain. Spine. 2013; 38(10):E609-E615;doi:10.1097/BRS.0b013e31828b2d09.

21. Elnaggar IM, Elhabashy HR, Abd El-Menam EM. Influence of spinal traction in treatment of cervical radiculopathy. Egypt J Neurol Psychiat Neurosurg. 2009;46(2): 455-461.

22. Bedwell C, Dowswell T, Neilson JP, Lavender T. The use of transcutaneous electrical nerve stimulation (TENS) for pain relief in labour: a review of the evidence. Midwifery. 2011;27(5):e141-e148; doi: 10.1016/j.midw.2009.12.004.

23. Borisut $S$, Vongsirinavarat $M$, Vachalathiti $R$, Sakulsriprasert $P$. Effects of strength and endurance training of superficial and deep neck muscles on muscle activities and pain levels of females with chronic neck pain. J Phys Ther Sci. 2013;25(9):1157-1162; doi: 10.1589/jpts.25. 1157.

24. Wade PG, Franklin CVJ. The effect of mobilization and core muscle strengthening for cervical spine in relieving cervicogenic headache. IOSR J Nurs Health Sci. 2015; 4(5):13-16; doi: 10.9790/1959-04511316.

25. Kumar D. Manual of Mulligan concept, $2^{\text {nd }}$ ed. New Delhi: Capri Institute of Manual Therapy; 2015.

26. Hing W, Hall T, Rivett D, Vicenzino B, Mulligan B. The Mulligan concept of manual therapy, $1^{\text {st }}$ ed. Chatswood: Churchill Livingstone; 2015.

27. Chettri P, Joshi N, Bista B. Effectiveness of neural mobilisation and intermittent cervical traction in cervical radiculopathy patients: a randomised clinical trial. SMU Med J. 2014;1(1):179-200.

28. Islam R, Quddus N, Miraj M, Anwer S. Efficacy of deep cervical flexor strength training versus conventional treatment in cervicogenic headache. Int J Cur Res Rev. 2013; 5(8):84-90.

29. Chiu TT, Ng JK, Walther-Zhang B, Lin RJ, Ortelli L, Chua SK. A randomized controlled trial on the efficacy of intermittent cervical traction for patients with chronic neck pain. Clin Rehabil. 2011;25(9):814-822; doi: 10.1177/ 0269215511399590.

30. Graham N, Gross AR, Goldsmith C. Mechanical traction for mechanical neck disorders: a systematic review. J Rehabil Med. 2006;38(3):145-152; doi: 10.1080/1650 1970600583029.

31. Hutton RS, Atwater SW. Acute and chronic adaptation of muscle proprioceptors in response to increased use. Sports Med. 1992;14(6):406-421; doi:10.2165/00007256$199214060-00007$.

32. Gross A, Kay TM, Paquin JP, Blanchette S, Lalonde P, Christie T, et al. Exercises for mechanical neck disorders. Cochrane Database Syst Rev. 2015;1:CD004250; doi: 10.1002/14651858.CD004250.pub5.

33. Verhagen AP, Karels C, Bierma-Zeinstra SM, Feleus A, Dahaghin S, Burdorf A, et al. Exercise proves effective in a systematic review of work-related complaints of the arm, neck, or shoulder. J Clin Epidemiol. 2007;60(2): 110-117; doi: 10.1016/j.jclinepi.2006.05.006.

34. Browder DA, Erhard RE, Piva SR. Intermittent cervical traction and thoracic manipulation for management of mild cervical compressive myelopathy attributed to cervical herniated disc: a case series. J Orthop Sports Phys Ther. 2004;34(11):701-712; doi: 10.2519/jospt.2004.34. 11.701. 
35. Gautam R, Dhamija JK, Puri A. Comparison of Maitland and Mulligan mobilization in improving neck pain, $\mathrm{ROM}$ and disability. Int J Physiother Res. 2014;2(3):482-487.

36. Ali H, Nasir RH, Hassan D. Effectiveness of cervical mobilization and cervical traction in management of non specific neck pain. J Riphah Coll Rehab Sci. 2015;3(2): 80-85.

37. Page P. Cervicogenic headaches: an evidence-led approach to clinical management. Int J Sports Phys Ther. 2011;6(3):254-266.

38. Gatterman MI. Standards of practice relative to complications of and contraindications to spinal manipulative therapy. J Can Chiropr Assoc. 1991;35(4):232-236.

39. Gibbons $P$, Tehan P. Spinal manipulation: indications, risks and benefits. J Bodyw Mov Ther. 2001;5(2):110119; doi: 10.1054/jbmt.2000.0208. 